\title{
Structural Response and Stochastic Impact Modeling
}

\author{
Renata Troian ${ }^{1, *}$, Manel Dallali ${ }^{1}$, Didier Lemosse ${ }^{1}$, and Leila Khalij $^{1}$ \\ ${ }^{1}$ Normandie universite, LMN/INSA de ROUEN, France
}

\begin{abstract}
The problem of the vulnerability of structures facing explosions came to the front line of the scientific scene in the last decades. Uncertainty of the environmental conditions and material properties have to be taken into account. The corresponding numerical models are very complex and depend on numerous parameters. Consequently, such models are cursed with issues which limit their use for real applications. Most of the existing approaches are based on a deterministic point of view, and are not able to represent the extreme sensitivity of a model towards uncertain parameters. That is why the uncertainty analysis is needed.

The proposed research is devoted to the analysis of a structural behavior under an uncertain impact loading. Elasto-plastic Bernoulli beam model is used as structural model for the case simplicity, while the different formulation for impact itself are studied to simulate the wide range of possible types of impact. Model sensitivity is studied first. The influence of input parameters on structural behavior, that are the impact force, duration and position, as well as beam material are then considered. The obtained insights can provide the guidelines for modeling the structure under the explosive loading taking into account the uncertainties.
\end{abstract}

\section{Introduction}

To assure the urban security efforts have to be made in developing reliability analysis and design methods. This analysis needs a detailed understanding of impact phenomena, structural and material response behavior of studied elements, namely metallic pipes, and uncertainty consideration. Research efforts, stimulated by industrial needs, are still required to achieve this goal.

The existing literature treats various aspects of the risks connected to the effects of explosions on structural integrity, but these works deal with the deterministic domain and studies concerning variability, reliability or uncertainty remain rare. Regarding the specific analysis of pipes or, more generally, cylindrical structures destined to the transportation of fluids, we may find works on the same topics, analogously limited to deterministic situations. Structural design by safety factors using nominal values without considering uncertainties may lead to designs that are either unsafe, or too conservative and thus not efficient.

While modeling the pipe's dynamic response one can't use anymore the material models developed for isotropic materials, as the pipe's construction material evolved much lately. Economic studies have shown that development of oil and gas transportation over long distances requires the use of high-strength grade steels because their tensile properties allow to substantially increase the internal pressure for a given pipe thickness [1]

In order to obtain high strength, these materials are produced using complex Thermo-Mechanical-ControlProcessing (TMCP) which introduces preferred orienta-

\footnotetext{
*e-mail: renata.troian@insa-rouen.fr
}

tions within the steel and leads to anisotropic plastic properties in higher grades. Rupture properties may also be anisotropic [2]. Fully understanding and describing the material behavior is needed to produce safe and costeffective pipelines.

Nevertheless, the pipe material properties can be controlled better comparing to possible debris' characteristics. Thus the attention have to be paid to a variable impactors modeling. Numerous experimental and numerical studies explosion (blast and impact of debris) on a cylinder have been carried out. But very few study concern the stochastic systems. Among them can be mentioned recent study of Wagner et al. [3] concerning robust design criterion for axially loaded cylindrical shells. Alizadeh et al. in [4] studied pipe conveying fluid with stochastic structural and fluid parameters.

We aim the present research to bring new knowledge on the subject and to deliver to scientific and industrial community new methods and models of explosion loading on metallic pipes taking into account uncertainties. For this we propose a simplified modeling of a pipe under an impact loading suitable for stochastic simulations. Impact is introduced into the model as a pulse of sinusoidal shape. First a sensitivity of a model versus loading parameters and pipe material is studied. Then a dynamic response of a pipe to several impacts is studied.

\section{Model of an impact on a metallic beam}

To simulate the impact several models can be used. Spring-mass models can be implemented for the determination of the varying contact force for the impacts on small specimens during the experiments for material properties 
tests. For the quasi-static systems it is possible to calculate the maximum contact force with energy-balance approach. It is assumed that when contact force reaches maximum the strain energy in the structure is equal to the kinetic energy of the projectile. When the dynamic of the structure have to be described more precisely, complete numerical models are in need. In this article we are interested in the response of the structure to the random impact. Thus there is a need of a simplified model, that includes a structure and numerous random impactors. The choice is to represent the impactors by the contact-force history, that act on the beam and to implement an elastic Bernoulli beam model pipe simulation. The characteristics of a perfectly clamped cylindrical steel beam are the following: length $l=1 \mathrm{~m}$, diameter $d=0.1 \mathrm{~m}$, module Young $E=2 e 11 \mathrm{~Pa}$ and density $\rho=7850 \mathrm{~kg} / \mathrm{m}^{3}$.

\subsection{Impactor impulse modeling}

The interest of the work is to study the response of the structure to stochastic impact loading, including the impact of solid debris and soft debris as well, as both types of the debris can be produced during an explosion and both can effect the structure's integrity. Two characteristics of the loading impulse are thus crucial - force and duration. To obtain the idea of the realistic contact force estimation for solid debris impact the paper of Perera et al. [5] can be considered. The model presented in this paper enables the value of the peak contact force generated by the impact of a piece of debris to be predicted. Results of calculations employing the derived relationships have been verified by comparison with experimental results across a wide range of impact scenarios. The observed contact load has sinusoidal shape, that will be considered in the present article. Duration time is the main difference between rigid and soft contacts, as are formulated in [6]. A comprehensive overview of soft impact models is done by Serge Abrate [7] for three types of soft projectile - liquid, bird and hailstone. Thus the rigid and soft impacts impulses are introduced into the model by considering impulse of different time duration.

Following [8] the sinusoidal impulse shape will be defined by its amplitude $h$ and duration $l$ by:

$$
\operatorname{load}=(\pi-2) h \sin (2 \omega t)
$$

for $0<t<\frac{\pi}{2 \omega}$, with $\omega=\frac{\pi-2}{l}$.

The developed numerical model of a beam under a impact is valid for the case of the elastic material, so the impactor's characteristics have to be in the ranges, which will provide the produced stresses in the beam that won't exceed the elastic limits that for a steel beam is $\sigma_{y}=2.5 e 8 P a$. Parametric study was conducted with varying amplitude and duration. Results presented in figure 1. The input parameters of the system that are considered to keep it in elastic domain are given in the Table 1.

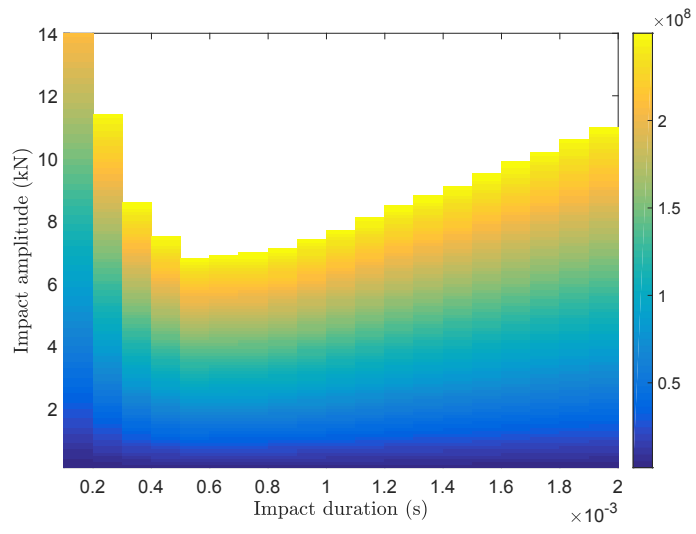

Fig. 1. Maximum stresses when impact is applied in the middle of beam $p=0.5$.

Table 1. Numerical values of parameters used in the simulation.

\begin{tabular}{lll} 
Input parameters & $\min$ & $\max$ \\
\hline Impact amplitude, $h, \mathrm{~N}$ & 0 & 6000 \\
Impact duration, $l, \mathrm{~s}$ & 0.001 & 0.02 \\
Impact position, $i_{p}, \mathrm{~m}$ & 0.05 & 0.5 \\
\hline
\end{tabular}

\section{Sensitivity analysis of the numerical model}

The sensitivity analysis has two main purposes. The first is to identify the input variables that have a strong influence on the output of the model. These variables have to be determined precisely to improve the accuracy of the model. The second aim is to identify, on the contrary, the one or more input variables that have less influence on the output. It is then not necessary to have a major precision for these variables. Thus, for models with a large number of inputs variables, the sensitivity analysis allows determining variables that have a significant impact on output and simplifies the model by neglecting the precision for input variables with small influence. Present section is devoted to the sensitivity analysis of the dynamic response of an elastic beam under a impact loading.

The input parameters are ranked according to their influence on the output. While there are many methods available for analyzing the decomposition of variance as a sensitivity measure, Sobol's method [9] is one of the most established and widely used methods and is capable of computing the Total Sensitivity Indices (TSI), which measures the main effects of a given parameter and all the interactions (of any order) involving that parameter.

\subsection{Parameters of the sensitivity study}

The study is concentrated around the stochastic nature of the impact load. Thus the principle parameters that will be studied will be the characteristics of the impact, such as the impact force, impact duration and impact position. The variability of the impacted structure, namely the Young's 
modulus $\mathrm{E}$ will be studied as well.

Parameters are supposed to be independent, distributed as random variables using Latin hypercube sampling (LHS) [10] in the limits given in the Table 1. The sample size is $N=1400$.

The output parameters of the model that are influenced by the impact characteristics and are important for the evaluation of the structure integrity are the beam deflection $w$ and stresses $\sigma$. Deflection and stresses won't be necessary maximum at the position of the impact, as it can be close to the clamped end of the beam. Thus we propose to consider them at the impact position and the maximum values on the whole length of the beam.

\subsection{Results}

Numerical results for sensitivity analysis of an elastic beam are obtained. First order effects and total effects of the stresses and beam deflection due to changes in loading characteristics were calculated. Numerical values are presented in the figure 2. It can be seen that the variation of Young's modulus doesn't play a major role in the obtained values. Contrariwise the position of the impact relatively to the boundary conditions and impact amplitude influence strongly the beam response. Impact duration variation affects the results as well.

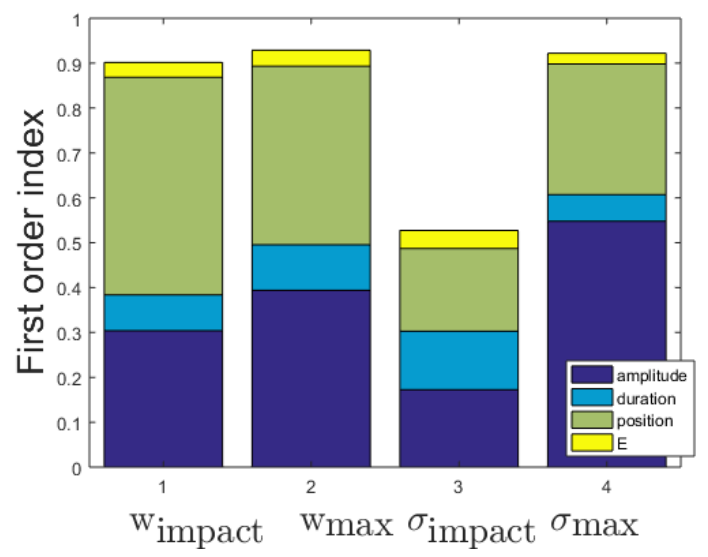

a)

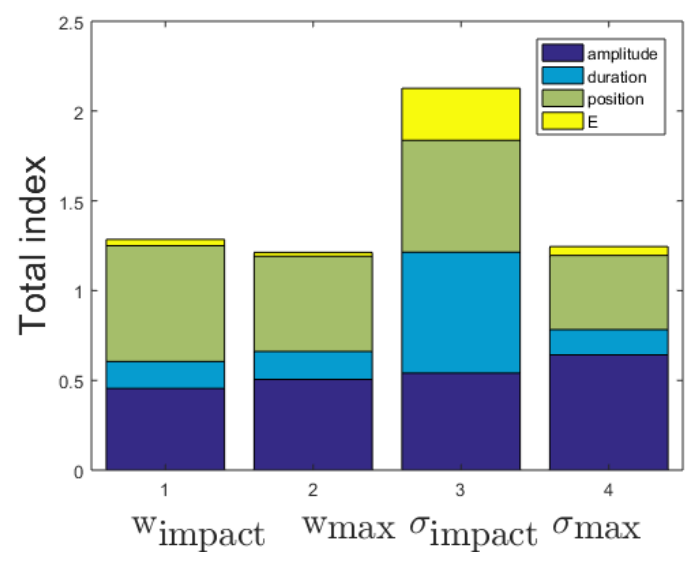

b)

Fig. 2. First (a) and total (b) indices using Sobol's method.

\section{Uncertainty analysis of an elastic model under multiply impact}

This section is devoted to uncertainty analysis of the structural response of an impacted beam. The realistic situation is modeled, when the possible probability of impactor is unknown but there is a need to compute the structural response. In this case the uniform distribution of the impulse parameters is used.

Situations of single impact, two impacts with different interval in between and three impacts with variable time were considered. Impulse characteristics, such as impact amplitude, duration and position (see Table 1) are considered as random variables with uniform distribution using Latin hypercube sampling (LHS). The sample size for single impact is $N=1000$, for two impacts $N=2000$ and for three $N=3000$. Obtained stresses distribution is presented in figures 3 and 4 . In red are marked number of tests when stresses exceeded the plastic limit $\sigma_{y}$.

Figures show, that even if the impactor's characteris-

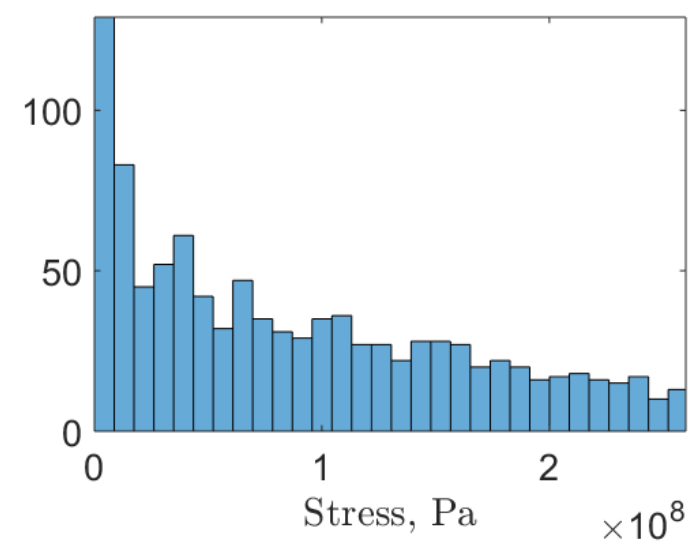

a)

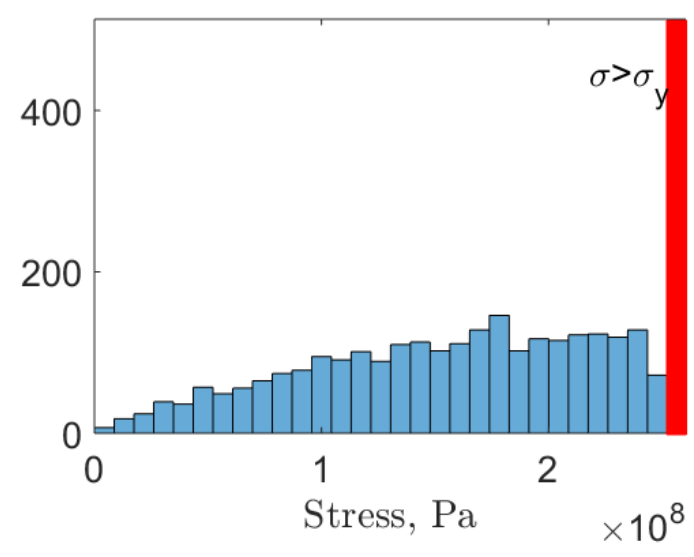

b)

Fig. 3. Distribution of possible stresses under (a) single impact (b) three impacts with the variable intervals between impacts.

tics don't lead to a plastic deformation in the case of single impact, two and more impactors of the comparable size and velocity can damage the impacted structure. Probability of stresses that reach $\sigma_{y}$ for the case of two impacts with an interval in between $0.001 \mathrm{~s}$ is $4 \%$, two impacts 


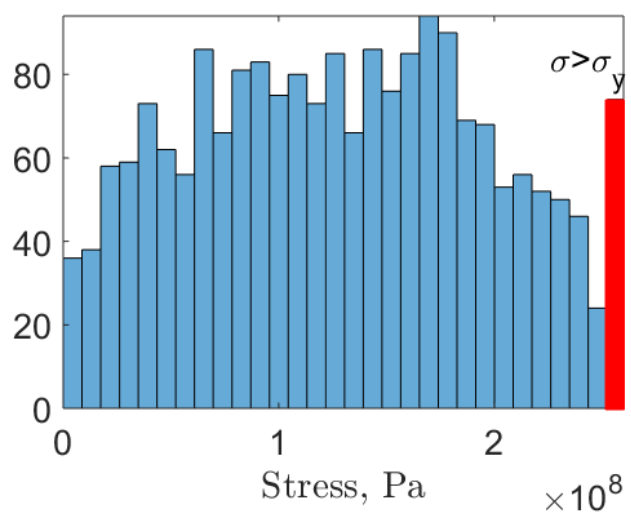

a)

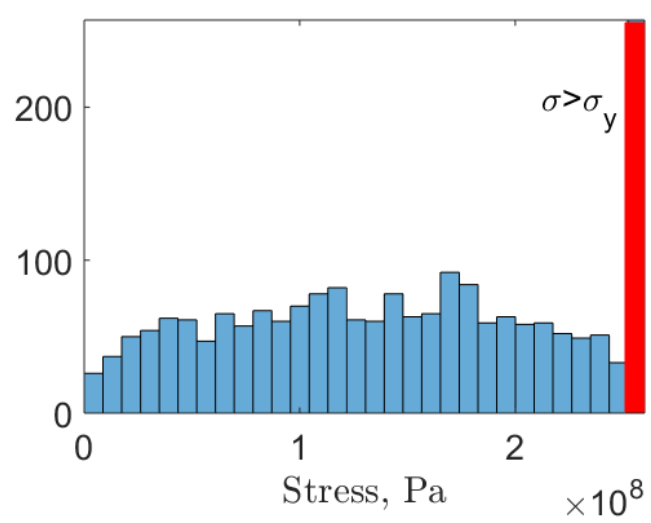

b)

Fig. 4. Distribution of possible stresses under two impacts with the interval of (a) $0.001 \mathrm{~s}$ and (b) $0.0005 \mathrm{~s}$.

with an interval $0.0005 \mathrm{~s}$ is $13 \%$, and for three impacts it goes up to $25 \%$.

\section{Further research and conclusions}

In the following, the elasto-plastic beam model was developed with LS-DYNA software to perform the uncertainty analysis in the plastic domain. The impactor is modeled as in the previous research by impulse shape.

A qualitative analysis of an impact was also conducted for validation of simplified simulations and understanding of a material response. A complete models of a steel pipe with variable impactors were developed with LS-DYNA. Steel, aluminum and concrete round impactors are considered.

This research is a part of a project AMED, that has been funded with the support from the European Union with the European Regional Development Fund (ERDF) and from the Regional Council of Normandie.

\section{References}

1. Y. Shinohara, Y. Madi, J. Besson, International Journal of Fracture 197, 127 (2016)

2. Y. Shinohara, Y. Madi, J. Besson, European Journal of Mechanics-A/Solids 29, 917 (2010)

3. H. Wagner, C. Hühne, S. Niemann, R. Khakimova, Thin-Walled Structures 115, 154 (2017)

4. A.A. Alizadeh, H.R. Mirdamadi, A. Pishevar, Engineering Structures 122, 24 (2016)

5. S. Perera, N. Lam, M. Pathirana, L. Zhang, D. Ruan, E. Gad, International Journal of Impact Engineering 91, 126 (2016)

6. U. Andreaus, P. Casini, Nonlinear Dynamics 22, 145 (2000)

7. S. Abrate, Progress in Aerospace Sciences 81, 1 (2016)

8. Y. Ren, X. Qiu, T. Yu, International Journal of Impact Engineering 70, 62 (2014)

9. I.M. Sobol, Mathematics and computers in simulation 55, 271 (2001)

10. M.D. McKay, R.J. Beckman, W.J. Conover, Technometrics 42, 55 (2000) 